\title{
VARIABILIDADE ESPACIAL DA INFILTRAÇÃO DE ÁGUA NO SOLO'1
}

\author{
CÍCERO AURÉLIO GRANGEIRO LIMA² e ALAIN PASSERAT DE SILANS ${ }^{3}$
}

\begin{abstract}
RESUMO - Dados de infiltração de água no solo foram obtidos em diversos pontos de uma malha retangular de uma parcela agrícola da Fazenda Experimental da EMEPA-PB, com o objetivo de caracterizar a variabilidade espacial da infiltração e dos parâmetros hidrodinâmicos do solo. Foram utilizadas as leis de Philip e de Green \& Ampt para ajustamento dos valores obtidos. Medições de granulometria, densidade do solo e umidade volumétrica antes e depois dos testes de infiltração foram efetuadas. Técnicas de análises estatísticas clássicas e geoestatísticas foram utilizadas para descrever a variabilidade espacial dos parâmetros de infiltração, obtidos pelo ajustamento às leis teóricas. Dos parâmetros estudados, o de sucção da equação de Green \& Ampt se mostrou o mais sensível à variabilidade espacial da parcela. Também não se notou correlação entre os parâmetros de infiltração e a textura.
\end{abstract}

Termos para indexação: textura, umidade do solo, geoestatística.

\section{SPATIAL VARIABILITY OF WATER INFILTRATION IN THE SOIL}

\begin{abstract}
Data of infiltration of water in soil were obtained in several points of a rectangular grid of an agricultural parcel of Experimental Farm of EMEPA-PB, Brazil, with the objective of characterizing the spatial variability of the infiltration and the hydrodynamic parameters of the soil. Data were fitted both to the Green \& Ampt and Philip equations for the adjustment of the values obtained. Granulometric, soil density and soil moisture measurements were performed just before and after the infiltration tests. Classical statistics and geostatistics were used to describe the spatial variability of infiltration parameters given by the fitting to the theoretical laws. Suction parameter of Green \& Ampt equation was shown to be the more sensitive to the soil spatial variability of the parcel. No correlation between infiltration parameter and soil texture was found.
\end{abstract}

Index terms: texture, soil moisture, geostatistics techniques.

\section{INTRODUÇÃO}

A estimativa da taxa de infiltração no solo é condicionante para determinar a repartição da precipitação em infiltração e escoamento superficial, a celeridade de penetração da frente de umidade no solo e a lâmina em projetos de irrigação. Por esses motivos, a utilização de infiltrômetros se popularizou na determinação das características hidrodinâmicas do solo relacionadas com a infiltração. Para esse propósito, utilizam-se geralmente duas

${ }^{1}$ Aceito para publicação em 31 de agosto de 1998.

${ }^{2}$ Eng. Civil, M.Sc., Programa de Desenvolvimento em Meio Ambiente da UFPB, Rua Cassimiro de Abreu 250, apto. 808, Jardim Luna, CEP 58033-330 João Pessoa, PB. E-mail: cdlima@netwaybbs.com.br

${ }^{3}$ Eng. Civil, Dr., Prof. Titular, Centro de Tecnologia, UFPB, CEP 58013-920 João Pessoa, PB. E-mail: alain@ct.ufpb.br equações de infiltração com forte embasamento físico: a equação de Green \& Ampt e a equação de Philip.

Embora não exista padronização dos sistemas de medição da infiltração, utilizam-se principalmente infiltrômetros duplo-anel ou simples-anel. A vantagem do primeiro sobre o segundo é de minimizar as infiltrações laterais, mantendo o fluxo na direção vertical. A sua desvantagem é uma operação mais complicada, principalmente porque necessita de maiores volumes de água.

Alguns estudos sobre variabilidade espacial das propriedades hidrodinâmicas do solo de parcelas agrícolas ou de vertentes de bacias hidrográficas (Rogowski, 1972; Nielsen et al., 1973; Carvallo et al., 1976; Sharma \& Luxmoore, 1979), indicaram que os solos, mesmo de aparência homogênea, apresentam considerável variabilidade, no espaço, das suas 
propriedades físicas. A consequiência dessa variabilidade sobre a resposta hidrológica de uma parcela não está ainda bem entendida (Sharma \& Luxmoore ,1979).

Neste trabalho, apresenta-se um estudo da variabilidade espacial das seguintes propriedades: a absorptividade, a condutividade hidráulica e a pressão capilar na frente de umidificação. Estas são determinadas pelo ajustamento das equações de Philip e de Green \& Ampt a valores experimentais. Testes de infiltração com infiltrômetro duplo-anel foram efetuados em uma parcela arenosa de $5.000 \mathrm{~m}^{2}$, de aparência homogênea situada na fazenda experimental da EMEPA (Empresa de Estudos e Pesquisas Agropecuárias do Estado da Paraíba), na cidade de João Pessoa. A variabilidade espacial é estudada através de uma análise estatística clássica, de uma análise de correlações e de uma análise geoestatística baseada na teoria das variáveis regionalizadas (Delhome, 1976).

Este trabalho teve como objetivo a caracterização da variabilidade espacial da infiltração e dos parâmetros hidrodinâmicos do solo das equações teóricas de Philip e de Green \& Ampt, utilizando-se técnicas de análises estatísticas clássicas e a geoestatística.

\section{MATERIAL E MÉTODOS}

\section{Desenvolvimento teórico}

As equações de Green \& Ampt e de Philip, que descrevem a evolução do volume infiltrado em função do tempo de infiltração, se escrevem, respectivamente, assim:

Equação de Green \& Ampt:

$\mathrm{I}=\mathrm{K}_{0} \mathrm{t}-\left(\mathrm{h}_{\mathrm{f}}-\mathrm{h}_{0}\right) \Delta \theta \ln \left(1-\frac{\mathrm{I}}{\Delta \theta\left(\mathrm{h}_{\mathrm{f}}-\mathrm{h}_{0}\right)}\right)$

Equação de Philip:

$\mathrm{I}=\mathrm{St}^{1 / 2}+\mathrm{At}$

Nessas equações, I representa a lâmina de água infiltrada no solo $(\mathrm{cm}) ; \mathrm{K}_{0}$, a condutividade hidráulica à saturação $(\mathrm{cm} / \mathrm{min}) ; \mathrm{h}_{\mathrm{f}}$ é a sucção na frente de umedecimento do solo (cm); $\mathrm{h}_{0}$ é a carga hidráulica acima do plano representado pela superfície do solo $(\mathrm{cm}) ; \Delta \theta=\theta_{\mathrm{S}}-\theta_{\mathrm{i}}\left(\mathrm{cm}^{3} / \mathrm{cm}^{3}\right)$ é a diferença entre a umidade volumétrica da frente de umidificação, $\theta_{\mathrm{S}}$, e a umidade volumétrica inicial do solo, $\theta_{\mathrm{i}} ; \mathrm{S}$ é a absorptividade $\left(\mathrm{cm} / \mathrm{min}^{1 / 2}\right)$; t é o tempo (min); e A é um parâmetro de ajuste com as mesmas dimensões que $\mathrm{K}_{0}(\mathrm{~cm} / \mathrm{min})$. O parâmetro A é freqüentemente confundido com a condutividade hidráulica à saturação $\mathrm{K}_{0}$, porém difere da mesma em função do teor volumétrico de ar que fica preso nos poros do solo.

Em virtude da inerente variabilidade espacial das propriedades hidrodinâmicas do solo, a aplicação simples das equações 1 e 2 não é satisfatória para caracterizar a infiltração numa determinada área, pois os processos físicos envolvidos não são lineares. A questão crucial para a irrigação ou para a hidrologia é o número muito elevado de medições que, teoricamente, devem ser feitas para calcular a infiltração média e/ou para caracterizar os parâmetros médios da infiltração, em uma parcela agrícola, de uma vertente ou de uma bacia hidrográfica. Se cada parâmetro for distribuído no espaço segundo uma lei de Gauss, o teorema-limite central permite calcular esse número. Outra questão que se coloca é saber se, uma vez identificada uma lei de distribuição desses parâmetros, tal lei se mantém ao longo do tempo.

A abordagem da questão da variabilidade espacial é estatística: 1) efetua-se a determinação dos parâmetros relativos às distribuições estatísticas de cada propriedade hidrodinâmica do solo para, através de simulações estocásticas, utilizando uma das equações de infiltração (equações 1 ou 2), gerar a função de distribuição da taxa de infiltração (Nielsen et al., 1973; Carvallo et al., 1976; Warrick et al., 1977; Sharma \& Luxmoore, 1979; Luxmoore \& Sharma, 1980; Russo \& Bresler, 1981). As propriedades hidrodinâmicas do solo envolvidas nas equações de infiltração, geralmente, não são estatisticamente independentes entre si, e as funções de distribuição de probabilidades conjuntas têm de ser determinadas. A teoria do fator de escala mostra-se atrativa para tentar diminuir o número de funções de distribuição a serem determinadas, e evitar a utilização das funções de distribuição de probabilidades conjuntas. Baseia-se na teoria da semelhança dos meios porosos (Miller \& Miller, 1956); 2) lança-se mão da análise geoestatística (Delhome, 1976). Observa-se que geralmente as propriedades hidrodinâmicas do solo não são distribuídas espacialmente de modo totalmente desordenado, mas apresentam certa estrutura que a abordagem estatística clássica (item i) não revela. Esta estrutura é identificada através do estudo da forma do semivariograma (Lima, 1992):

$\gamma(\mathrm{h})=\frac{1}{2} \operatorname{var}\{\mathrm{Z}(\mathrm{x}+\mathrm{h})-\mathrm{Z}(\mathrm{x})\}$ 
onde $\mathrm{Z}$ representa a propriedade do solo analisada, considerada como uma variável aleatória, função da posição da amostragem $\mathrm{x}$, e $\mathrm{h}$ é a distância entre dois pontos de amostragem.

Basicamente, a estrutura espacial é analisada dependendo do comportamento do semivariograma na origem e no infinito. Na origem (Fig. 1), quatro aspectos são possíveis: o aspecto parabólico, que traduz uma variável muito regular; o aspecto linear, que corresponde a uma variável menos regular; o efeito de pepita (nugget effect), que traduz um efeito muito irregular (dois pontos distintos, porém vizinhos, podem apresentar valores bem diferentes. Tal irregularidade pode ser devida a uma microrregionalização de escala inferior à escala de amostragem); $\mathrm{e}$ $\mathrm{o}$ aspecto aleatório puro, que traduz a inexistência de correlação entre pontos. Ao infinito (Fig. 2), duas situações são possíveis: variograma ao infinito limitado, e variograma ao infinito com patamar e portança. Ao contrário do variograma não-limitado (indicativo de deriva), aquele que apresenta um patamar indica que nas distâncias entre pontos de amostragem superior à portança, os valores da propriedade não são correlatos entre si. Nesses casos, o valor do patamar corresponde à variança espacial da amostra.

Na Tabela 1, apresenta-se uma relação não-exaustiva de parâmetros estatísticos característicos da variabilidade espacial da infiltração, deduzidos de estudos efetuados nas décadas de 70 e 80 . As áreas de estudos vão da pequena parcela agrícola à bacia hidrográfica, onde os estudos realizados em uma bacia hidrográfica são relativamente

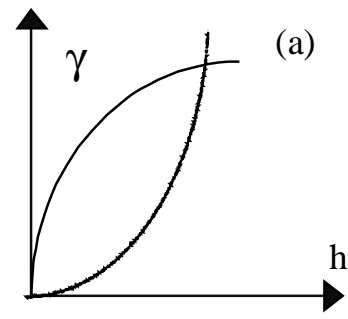

(c)

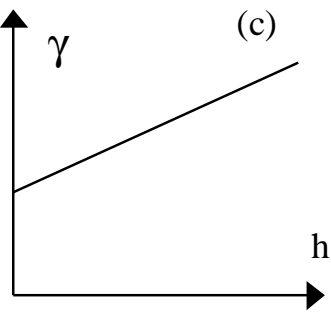

FIG.1. Variogramas na origem: (a) aspecto parabólico, (b) aspecto linear, (c) aspecto aleatório com efeito de pepita, (d) aspecto aleatório puro. poucos, quando comparados com os realizados em parcelas agrícolas.

\section{Estudo experimental}

A parcela escolhida para o estudo situa-se na Fazenda Experimental da EMEPA, em João Pessoa. Nessa, foi demarcada uma área de $5.000 \mathrm{~m}^{2}$, na qual foi traçada uma malha retangular regular com espaçamentos de $15 \mathrm{~m}$, totalizando 32 nós. Em cada nó foram efetuados testes de infiltração com duração de 90 minutos, com infiltrômetro duplo-anel de carga constante. Foram determinadas, pelo o método gravimétrico-padrão, as umidades volumétricas de amostras coletadas antes e após o teste de infiltração. Também foi determinado o peso específico aparente do solo seco, a partir de amostras indeformáveis nos 20 primeiros centímetros do solo. Em cada ponto de medição foi também efetuada uma análise granulométrica, e determinada a porcentagem da fração de argila + silte. Maiores detalhes sobre o estudo experimental encontram-se em Lima (1992).

\section{RESULTADOS E DISCUSSÃO}

$\mathrm{Na}$ Tabela 2, encontram-se os valores dos parâmetros relativos às equações 1 e 2 , obtidos em cada nó da malha de estudo. Os valores experimentais de $\mathrm{S}$ e A, que constam da Tabela 2, foram obtidos pelo ajustamento da equação 2 aos valores de I $(\mathrm{t})$, por uma regressão linear simples entre o produto $\mathrm{I}^{-1 / 2} \mathrm{e} \mathrm{t}^{1 / 2}$. O valor da absorptividade obtido no ponto 29 é criticável, visto que a sorptividade não pode ser negativa. A explicação mais provável está na existência de infiltrações preferenciais em macroporos ou ao longo de raízes.

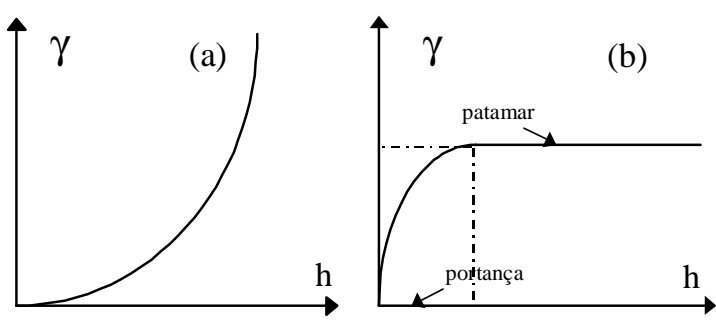

FIG. 2. Variogramas ao infinito: (a) não-limitado, (b) com patamar e portança.

Pesq. agropec. bras., Brasília, v.34, n.12, p.2311-2320, dez. 1999 
TABELA 1. Variabilidades de alguns parâmetros do solo.

\begin{tabular}{|c|c|c|c|c|c|c|c|c|}
\hline Parâmetro & $\begin{array}{c}\text { Função de } \\
\text { distribuição }\end{array}$ & $\mu$ & $\sigma$ & $\begin{array}{l}\mathrm{CV} \\
(\%)\end{array}$ & Área & $\begin{array}{c}\mathrm{N}^{\circ} \mathrm{de} \\
\text { medições }\end{array}$ & $\begin{array}{l}\text { Método } \\
\text { utilizado }\end{array}$ & Referência \\
\hline $\mathrm{S}\left(\mathrm{cm} / \mathrm{h}^{1 / 2}\right)$ & Log-Normal & 4,95 & 2,44 & 49,0 & & & Duplo & Sharma et al. (1980) \\
\hline $\mathrm{A}(\mathrm{cm} / \mathrm{h})$ & Log-Normal & 1,46 & 0,22 & 60,0 & 9,6 ha & 26 & Anel & \\
\hline $\mathrm{S}\left(\mathrm{cm} / \mathrm{h}^{1 / 2}\right)$ & Normal & 11,04 & 0,84 & 7,6 & 0,8 ha & 30 & $\begin{array}{l}\text { Simples } \\
\text { Anel }\end{array}$ & $\begin{array}{c}\text { Russo \& Bresler } \\
\text { (1980) }\end{array}$ \\
\hline $\mathrm{S}\left(\mathrm{cm} / \mathrm{h}^{1 / 2}\right)$ & Normal & 9,35 & 3,20 & 34,0 & & & Duplo & Vauclin et al. (1981) \\
\hline $\mathrm{A}(\mathrm{cm} / \mathrm{h})$ & Normal & 14,30 & 3,80 & 26,0 & 1,0 ha & 19 & Anel & \\
\hline $\mathrm{K}_{0}(\mathrm{~cm} / \mathrm{h})$ & Log-Normal & 0,27 & 0,19 & 71,0 & & 625 & & Sisson \& Wierenga \\
\hline $\mathrm{K}_{0}(\mathrm{~cm} / \mathrm{h})$ & Log-Normal & 0,35 & 0,19 & 56,0 & $40 \mathrm{~m}^{2}$ & 125 & Simples & $(1981)$ \\
\hline $\mathrm{K}_{0}(\mathrm{~cm} / \mathrm{h})$ & Log-Normal & 0,36 & 0,08 & 22,0 & & 25 & Anel & \\
\hline $\mathrm{K}_{0}(\mathrm{~cm} / \mathrm{h})$ & Normal & 0,69 & 0,28 & 40,0 & 0,9 ha & 1280 & $\begin{array}{l}\text { Simples } \\
\text { Anel }\end{array}$ & Vieira et al. (1981) \\
\hline $\mathrm{K}_{0}(\mathrm{~cm} / \mathrm{h})$ & Log-Normal & 0,02 & 0,11 & 130 & - & - & - & $\begin{array}{c}\text { Luxmoore \& Sharma } \\
\text { (1980) }\end{array}$ \\
\hline
\end{tabular}

Os valores da condutividade na saturação natural relativos aos 32 pontos da parcela foram obtidos a partir das declividades da assíntota das curvas de infiltração acumulada.

A sucção capilar $h_{f}$ foi determinada em cada ponto utilizando a equação 1 . A solução desta equação foi obtida pelo método iterativo de NewtonRaphson. A equação de Green \& Ampt supõe uma distribuição vertical uniforme da umidade relativa préinfiltração. Ela permite calcular o volume infiltrado I, considerando também que a frente de umidificação permanece plana e horizontal ao longo do teste. Essas hipóteses não são sempre respeitadas, o que gerou valores coerentes de $\mathrm{h}_{\mathrm{f}}$ em somente 13 pontos. $\mathrm{O}$ valor de $\mathrm{h}_{\mathrm{f}}$ é teoricamente dependente da umidade inicial do solo, embora Clapp \& Hornberger (1978) tenham proposto valores típicos, tendo em vista uma classificação textural do solo. Considerando a possibilidade de uma distribuição vertical nãouniforme da umidade inicial, temos calculado valores de $h_{f}$ para os tempos de infiltração de 30 e 90 minutos.

\section{Estudo estatístico clássico}

Os valores da média, desvio-padrão, coeficiente de variação, coeficiente de assimetria e curtose foram calculados em relação a todos os parâmetros considerados, e procurou-se ajustar os dados às leis de distribuição Normal ou Log-Normal, com nível de significância inferior ou igual a 5\%, escolhen- do a distribuição de freqüência que melhor se ajusta. Os resultados aparecem na Tabela 3. Constata-se que $h_{f}$ não se ajusta a nenhuma das duas leis de distribuição testadas. Além do mais, este parâmetro é que mais varia espacialmente. Todos os outros parâmetros se ajustaram à lei de distribuição Normal com nível de significância inferior ou igual a $5 \%$. Comparando-se a variabilidade espacial apresentada nesta experiência com as variabilidades que constam da Tabela 1, nota-se uma variabilidade espacial razoável. Os valores de $\mathrm{K}_{0}, \mathrm{~S}$ e do teor percentual em argila e silte apresentam um coeficiente de variação pouco inferior a $50 \%$.

Vachaud et al. (1985) têm mostrado que em três sítios diferentes nos quais trabalharam (França, Tunísia e Senegal), as funções de distribuição do teor em água no solo permanecem estáveis ao longo do tempo. Embora não existam, até o presente momento, outros estudos desta natureza, tentando comprovar a universalidade dessa regra, os resultados obtidos sugerem certo grau de determinismo na variabilidade espacial. Assim, torna-se necessário procurar observar se os parâmetros considerados apresentam correlação com a textura através do seu conteúdo em partículas finas (percentual de argila + silte) ou com a sua posição dentro da parcela.

\section{Análise de correlações}

Os coeficientes de correlação obtidos a partir da análise de correlação simples entre a taxa percentual da fração de argila + silte e as demais variáveis das 
TABELA 2. Valores dos parâmetros de infiltração para os 32 nós da grade regular¹

\begin{tabular}{|c|c|c|c|c|c|c|c|c|c|c|}
\hline Ponto & $\begin{array}{c}\theta_{\mathrm{S}} \\
\left(\mathrm{cm}^{3} / \mathrm{cm}^{3}\right)\end{array}$ & $\begin{array}{c}\theta_{\mathrm{i}} \\
\left(\mathrm{cm}^{3} / \mathrm{cm}^{3}\right)\end{array}$ & $\begin{array}{c}\Delta \theta \\
\left(\mathrm{cm}^{3} / \mathrm{cm}^{3}\right)\end{array}$ & $\begin{array}{c}\text { Argila }+ \text { Silte } \\
(\%)\end{array}$ & $\begin{array}{c}\rho_{\mathrm{S}} \\
\left(\mathrm{g} / \mathrm{cm}^{3}\right)\end{array}$ & $\begin{array}{c}\mathrm{S} \\
\left(\mathrm{cm} / \min ^{1 / 2}\right)\end{array}$ & $\begin{array}{c}\mathrm{A} \\
(\mathrm{cm} / \mathrm{min})\end{array}$ & $\begin{array}{c}\mathrm{K}_{0} \\
(\mathrm{~cm} / \mathrm{min})\end{array}$ & $\begin{array}{l}\mathrm{h}_{\mathrm{f}} 30 \\
(\mathrm{~cm})\end{array}$ & $\begin{array}{l}\mathrm{h}_{\mathrm{f}} 90 \\
(\mathrm{~cm})\end{array}$ \\
\hline 01 & 0,17 & 0,05 & 0,12 & 1,33 & 1,15 & 0,389 & 0,287 & 0,350 & - & - \\
\hline 02 & 0,26 & 0,06 & 0,20 & 1,57 & 1,38 & 0,696 & 0,428 & 0,500 & - & - \\
\hline 03 & 0,25 & 0,05 & 0,20 & 1,48 & 1,49 & 0,539 & 0,492 & 0,525 & 1,11 & 1,02 \\
\hline 04 & 0,26 & 0,06 & 0,20 & 1,34 & 1,42 & 0,440 & 0,695 & 0,700 & 0,87 & 1,69 \\
\hline 05 & 0,24 & 0,11 & 0,13 & 2,97 & 1,27 & 0,519 & 0,218 & 0,280 & 2,28 & 0,82 \\
\hline 06 & 0,25 & 0,07 & 0,18 & 2,88 & 1,37 & 0,328 & 0,421 & 0,500 & - & - \\
\hline 07 & 0,26 & 0,06 & 0,20 & 1,41 & 1,44 & 0,917 & 0,578 & 0,6 & 5,88 & 7,16 \\
\hline 08 & 0,25 & 0,08 & 0,17 & 2,46 & 1,40 & 1,58 & 0,464 & 0,600 & - & - \\
\hline 09 & 0,29 & 0,06 & 0,23 & 2,15 & 1,35 & 0,816 & 0,009 & 0,025 & 51,89 & 48,96 \\
\hline 10 & 0,30 & 0,05 & 0,25 & 2,18 & 1,49 & 0,772 & 0,044 & 0,100 & 7,71 & 5,24 \\
\hline 11 & 0,21 & 0,04 & 0,17 & 1,71 & 1,32 & 1,292 & 0,509 & 0,600 & - & - \\
\hline 12 & 0,28 & 0,04 & 0,24 & 2,92 & 1,37 & 0,372 & 0,660 & 0,600 & 1,79 & 5,19 \\
\hline 13 & 0,26 & 0,08 & 0,18 & 2,43 & 1,39 & 0,436 & 0,668 & 0,650 & 1,58 & 3,66 \\
\hline 14 & 0,25 & 0,06 & 0,19 & 3,13 & 1,42 & 0,492 & 0,347 & 0,400 & - & - \\
\hline 15 & 0,22 & 0,09 & 0,13 & 2,57 & 1,33 & 0,610 & 0,335 & 0,500 & - & - \\
\hline 16 & 0,30 & 0,05 & 0,25 & 2,80 & 1,41 & 1,011 & 0,531 & 0,450 & 12,39 & 21,34 \\
\hline 17 & 0,25 & 0,07 & 0,18 & 3,39 & 1,40 & 0,360 & 0,286 & 0,300 & 0,53 & 0,81 \\
\hline 18 & 0,23 & 0,06 & 0,17 & 3,16 & 1,42 & 0,300 & 0,447 & 0,500 & - & - \\
\hline 19 & 0,27 & 0,05 & 0,17 & 2,59 & 1,35 & 0,528 & 0,650 & 0,700 & 1,02 & 0,52 \\
\hline 20 & 0,27 & 0,05 & 0,22 & 3,00 & 1,42 & 0,818 & 1,050 & 0,800 & - & - \\
\hline 21 & 0,25 & 0,06 & 0,19 & 5,25 & 1,39 & 0,237 & 0,271 & 0,300 & - & - \\
\hline 22 & 0,23 & 0,07 & 0,16 & 6,18 & 1,36 & 0,070 & 0,347 & 0,300 & - & - \\
\hline 23 & 0,29 & 0,05 & 0,24 & 5,0 . & 1,14 & 1,800 & 0,655 & 0,800 & - & - \\
\hline 24 & 0,24 & 0,05 & 0,19 & 4,43 & 1,39 & 0,921 & 0,800 & 0,500 & 3,26 & 5,79 \\
\hline 25 & 0,25 & 0,07 & 0,18 & 6,26 & 1,33 & 0,249 & 0,289 & 0,300 & - & - \\
\hline 26 & 0,19 & 0,05 & 0,14 & 4,72 & 1,25 & 0,955 & 0,573 & 0,600 & 4,98 & 6,56 \\
\hline 27 & 0,20 & 0,05 & 0,15 & 6,33 & 1,12 & 0,342 & 0,867 & 0,900 & - & - \\
\hline 28 & 0,25 & 0,05 & 0,20 & 3,88 & 1,40 & 0,521 & 0,350 & 0,600 & - & - \\
\hline 29 & 0,25 & 0,05 & 0,20 & 4,63 & 1,39 & $-0,132$ & 0,458 & 0,600 & - & - \\
\hline 30 & 0,27 & 0,05 & 0,22 & 4,91 & 1,36 & 0,233 & 0,554 & 0,550 & - & - \\
\hline 31 & 0,23 & 0,05 & 0,18 & 4,95 & 1,21 & 0,395 & 0,449 & 0,500 & - & - \\
\hline 32 & 0,27 & 0,06 & 0,21 & 3,79 & 1,27 & 0,190 & 0,751 & 0,850 & - & - \\
\hline Média & 0,25 & 0,06 & 18,84 & 3,26 & 1,35 & 5,20 & 0,484 & 0,515 & 7,33 & 8,37 \\
\hline Desv. Pad & 0,03 & 0,015 & 3,62 & 1,57 & 0,093 & 2,53 & 0,229 & 0,202 & 13,82 & 13,37 \\
\hline
\end{tabular}

$\theta_{\mathrm{S}}$ : teor de umidade volumétrica do solo após o teste de infiltração; $\mathrm{q}_{\mathrm{i}}$ : teor de umidade volumétrica do solo antes do teste de infiltração; $\Delta \theta$ : variação do teor de umidade volumétrica; $\mathrm{S}$ : absortividade; A: constante da equação de Philip; $\mathrm{K}_{0}$ : condutividade hidráulica no regime permanente de infiltração; $\mathrm{r}_{\mathrm{S}}$ : peso específico do solo seco; $\mathrm{h}_{\mathrm{f}}$ : sucção capilar.

TABELA 3. Ajustamento à função de distribuição estatística.

\begin{tabular}{ccccccc}
\hline Parâmetros & $\begin{array}{c}\text { Função de } \\
\text { distribuição }\end{array}$ & $\mu$ & $\sigma$ & CV (\%) & Assimetria & Curtose \\
\hline$\theta_{\mathrm{S}}$ & Normal & 24,57 & 3,03 & 12,13 & $-0,65$ & 3,88 \\
$\Delta \theta$ & Normal & 18,84 & 3,62 & 19,18 & $-0,04$ & 2,85 \\
$\%$ (Argila + Silte) & Normal & 3,26 & 1,57 & 48,07 & 0,51 & 2,53 \\
$\mathrm{~S}$ & Normal & 5,20 & 2,53 & 48,68 & 0,49 & 2,55 \\
$\mathrm{~A}$ & Normal & 0,484 & 0,229 & 47,28 & 0,19 & 3,54 \\
$\mathrm{~K}_{0}$ & Normal & 0,504 & 0,208 & 41,23 & $-0,24$ & 2,80 \\
$\mathrm{~h}_{\mathrm{f}}$ & - & 26,77 & 64,51 & 242,51 & - & - \\
\hline
\end{tabular}

equações de infiltração na parcela estudada constam da Tabela 4.

Os valores apresentados nessa tabela mostram que não existem correlações significativas entre o fator textural representado pela porcentagem da fração de argila + silte e as propriedades hidrodinâmicas do solo. Imbernon (1981) chega às mesmas conclusões num experimento desenvolvido no Senegal, em uma parcela com predominância de solo arenoso.

Através da análise de correlação múltipla, procurou-se detectar a existência de uma possível direção privilegiada das propriedades físicas e 
hidrodinâmicas do solo. Escolheu-se um sistema de referência ortogonal cujo eixo das ordenadas é orientado na direção longitudinal da malha. Chamando-se de W a variável dependente, ou seja, a propriedade analisada, procurou-se estabelecer a relação linear:

$$
\mathrm{W}=\mathrm{aX}+\mathrm{bY}+\mathrm{c},
$$

calculando-se os coeficientes de correlação múltipla e parciais. Os resultados constam da Tabela 5, onde, $\mathrm{R}$ representa o coeficiente de correlação múltipla; $\mathrm{r}_{X}$ e $r_{Y}$ são os coeficientes de correlação, com as direções X e Y, respectivamente; $s_{R}$ representa o desviopadrão dos resíduos, e $\% \mathrm{~s}^{2}$ é a porcentagem de variância explicada pelas coordenadas X e Y.

Os resultados desta tabela mostram que $67,7 \%$ da variância da fração de argila + silte na parcela é explicada pela posição do ponto de amostragem. Além do mais, o teor de argila + silte é fortemente correlato com o eixo Y, isto é, com a direção longitu-

TABELA 4. Coeficientes de correlação simples entre a taxa percentual da fração (argila + silte) e as demais variáveis.

\begin{tabular}{ccc}
\hline $\begin{array}{c}\text { Variável } \\
\text { dependente }\end{array}$ & $\begin{array}{c}\text { Variável } \\
\text { independente }\end{array}$ & $\begin{array}{c}\text { Coeficiente de } \\
\text { correlação }\end{array}$ \\
\hline & $\theta_{\mathrm{S}}\left(\mathrm{cm}^{3} / \mathrm{cm}^{3}\right)$ & $-0,16$ \\
$\%$ (argila + silte) $)$ & $\mathrm{S}\left(\mathrm{cm}^{1 / 2}\right)$ & $-0,32$ \\
& $\mathrm{~A}\left(\mathrm{~cm} / \mathrm{min}^{1 / 2}\right)$ & $+0,09$ \\
& $\mathrm{~K}_{0}(\mathrm{~cm} / \mathrm{min})$ & $-0,02$ \\
& $\Delta \theta\left(\mathrm{cm}^{3} / \mathrm{cm}^{3}\right)$ & $-0,09$ \\
\hline
\end{tabular}

dinal da parcela. Aproximadamente $35 \%$ das variâncias respectivas de $\mathrm{A}_{\text {e }} \mathrm{K}_{0}$ são explicadas pela posição do ponto de amostragem, porém sem a indicação de uma direção privilegiada. Esses resultados confirmam a inexistência de correlações entre o teor de argila + silte e as demais variáveis encontradas a partir dos resultados da Tabela 4 .

\section{Análise geoestatística}

A existência da estrutura espacial e sua caracterização, caso exista, foi feita a partir da análise dos semivariogramas. Dois casos foram considerados: a análise dos semivariogramas reduzidos brutos, e a análise dos semivariogramas reduzidos filtrados.

O semivariograma reduzido corresponde ao gráfico $\frac{\gamma}{\sigma^{2}}=\mathrm{f}(\mathrm{h})$ (equação 4 ), onde $\mathrm{s}^{2}$ é a variância da variável aleatória considerada. Os semivariogramas reduzidos brutos correspondendo às varáveis $\%$ (argila + silte), $\mathrm{S}, \theta_{\mathrm{S}}, \mathrm{A}, \mathrm{K}_{0}$ e $\Delta \theta$ são representados na Fig. 3. Destes, os relativos às variáveis $S, \theta_{\mathrm{S}}$ e $\Delta \theta$ correspondem a uma distribuição aleatória pura. $\mathrm{O}$ semivariograma relativo à variável \% (argila + silte) é de forma parabólica, com possível efeito de pepita na origem, e não-limitado ao infinito. Considerando a forte correlação desta variável com a posição do ponto de amostragem na parcela, e principalmente com a direção transversal, define-se uma variável aleatória residual filtrando esta dependência espacial determinista. O mesmo, embora em escala muito menor, é feito com as variáveis $\mathrm{A}$ e $\mathrm{K}_{0}$.

TABELA 5. Coeficientes de correlação múltipla1

\begin{tabular}{crrrrrrrr}
\hline $\begin{array}{c}\text { Variável } \\
\text { dependente }\end{array}$ & $\mathrm{a}$ & $\mathrm{b}$ & $\mathrm{c}$ & $\mathrm{R}$ & $\mathrm{r}_{\mathrm{X}}$ & $\mathrm{r}_{\mathrm{Y}}$ & $\sigma_{\mathrm{R}}$ & $\% \sigma^{2}$ \\
\hline$\%$ (argila + silte) & $-0,01$ & 0,04 & 1,35 & 0,82 & $-0,13$ & 0,82 & 0,85 & 67,70 \\
$\theta_{\mathrm{S}}$ & 0,00 & 0,00 & 0,24 & 0,35 & 0,28 & $-0,23$ & 0,03 & 12,22 \\
$\mathrm{~S}$ & 0,09 & $-0,02$ & 3,94 & 0,43 & 0,40 & $-0,19$ & 0,03 & 18,50 \\
$\mathrm{~A}$ & 0,09 & 0,02 & 1,50 & 0,59 & 0,54 & 0,34 & 0,18 & 34,69 \\
$\mathrm{~K}_{0}$ & 0,09 & 0,01 & 2,17 & 0,60 & 0,57 & 0,26 & 0,15 & 35,80 \\
$\Delta \theta$ & 0,00 & 0,00 & 0,17 & 0,30 & 0,29 & $-0,10$ & 0,03 & 0,22 \\
\hline
\end{tabular}

${ }^{1}$ a, b e c: coeficente de correlações parciais; R: coeficiente de correlação múltipla; $r_{\mathrm{x}}$ e $r_{\mathrm{y}}$ : coeficiente de correlação com as direções x e y; $\sigma_{\mathrm{R}}$ : desviopadrão dos resíduos; $\% \mathrm{~s}^{2}$ : porcentagem das variâncias explicada pelas coordenadas x e y; $\theta_{\mathrm{S}}$ : teor de umidade do solo, após o teste de infiltração; S: absortividade; A: constante da equação de Philip; $K_{0}$ : condutividade hidráulica na superfície em regime permanente de infiltração. $\Delta \theta$ : variação do teor de umidade volumétrica. 
Na Fig. 4, são representados os semivariogramas reduzidos filtrados correspondentes às variáveis $\%$ (argila + silte), A e $\mathrm{K}_{0}$. Os melhores ajustamentos encontrados neles correspondem a ajustamentos lineares. Nota-se que na variável \% $(\mathrm{A}+\mathrm{S})$, um patamar se desenvolve a partir de uma distância da ordem de $15 \mathrm{~m}$. Isso significa que os resíduos desta variável, uma vez filtradas as dependências com as coordenadas e principalmente com a coordenada Y, não são mais correlatas a partir de uma distância (portança) de $15 \mathrm{~m}$. Observa-se, também, que a reta indicativa deste patamar se encontra acima do valor um, valor para o qual $\gamma(\mathrm{h})$ é constante e igual à variância da amostra. Parte da variância deve ser atribuída à existência de uma estrutura em distâncias inferiores a $15 \mathrm{~m}$. O semivariograma deve portanto apresentar um efeito de pepita, que poderia ser caracterizado melhor se medições com espaçamentos inferiores a $15 \mathrm{~m}$ tivessem sido realizadas. No caso das variáveis $\mathrm{A}$ e $\mathrm{K}_{0}$, a reta de ajustamento possui declividade.
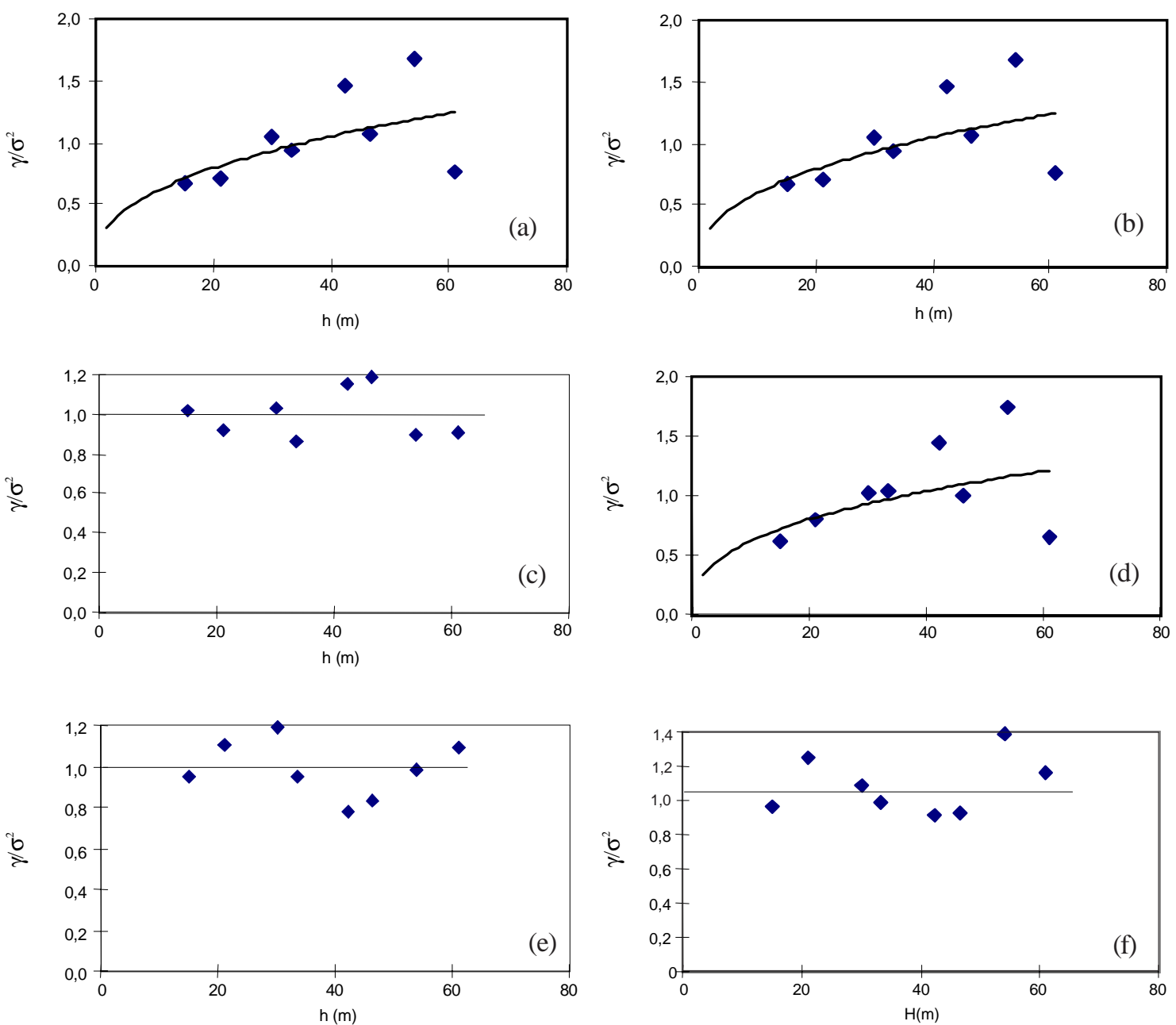

FIG. 3. Semivariogramas reduzidos brutos: (a) \% (argila + silte); (b) constante da equação de Philip; (c) absortividade; (d) condutividade hidráulica; (e) umidade volumétrica após teste de infiltração; (f) variação do teor de umidade volumétrica. 

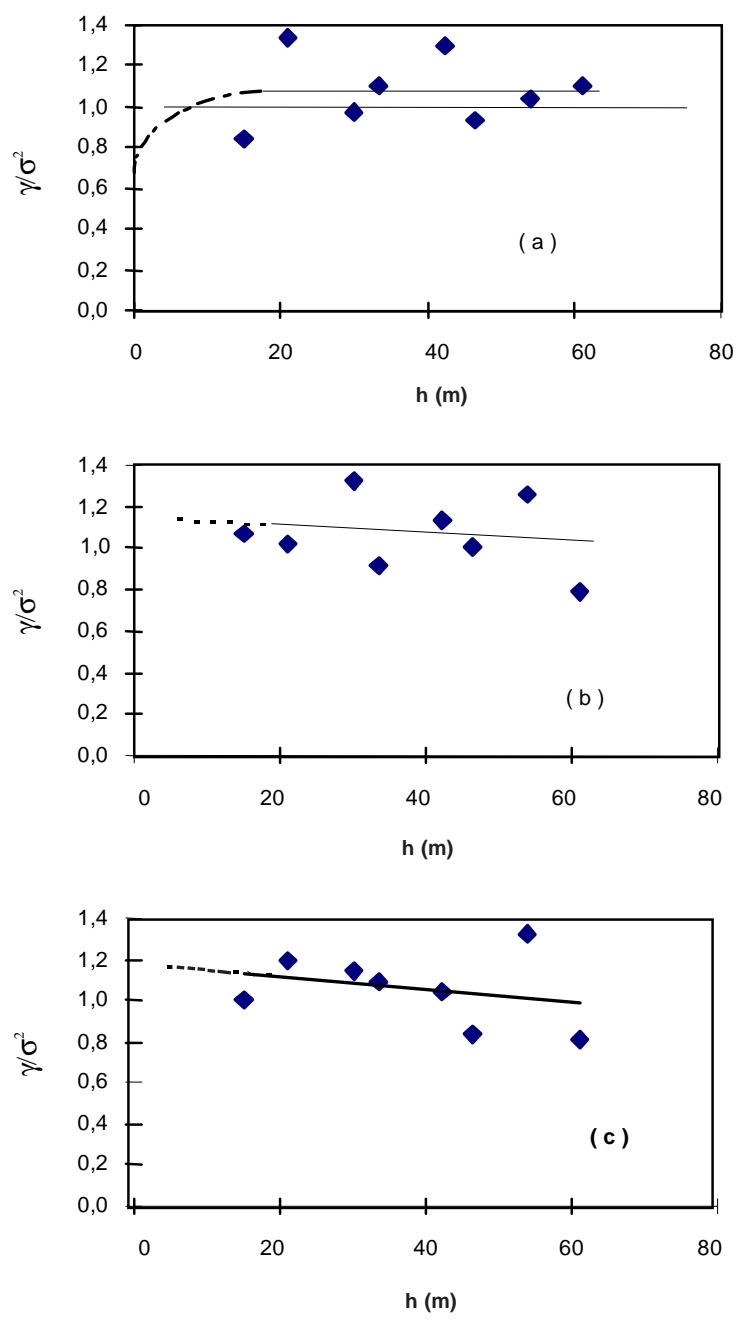

FIG.4. Semivariogramas reduzidos filtrados dos parâmetros: (a) \% (argila+silte); (b) constante da equação de Philip; (c) condutividade hidráulica.

O resultado da consideração da deriva explica apenas parcialmente a variância espacial da amostra, pela posição dos pontos no plano horizontal da parcela. O resto da variância deve ser explicado também por um efeito de pepita, tal como sugerido pelos semivariogramas brutos.

A variabilidade espacial da parcela considerada na fazenda da EMEPA (aproximadamente $5.000 \mathrm{~m}^{2}$ ) foi caracterizada através de análises estatísticas. Tal caracterização requer um número importante de medições. Correlações entre textura, através da taxa percentual da fração de argila + silte, e os parâmetros hidrodinâmicos, se mostraram insignificantes, motivo pelo qual inferir esses parâmetros por uma análise mais simples de granulometria não foi, no solo estudado, possível. Resultados similares foram encontrados por Imbernon (1981), numa parcela de solo também arenoso no Senegal.

Constata-se que na equação de infiltração de Philip (equação 2), os coeficientes S e A são constantes ao longo do tempo, o mesmo não acontecendo com a equação de Green \& Ampt (equação 1). O volume infiltrado médio, na parcela, em função do tempo, pode ser obtido pela equação de Philip, considerando valores médios, na parcela, da absorptividade $\mathrm{S}$ e do parâmetro A. A questão que se coloca então é localizar, na parcela, o ponto onde se encontram esses valores médios. A mesma constatação se faz quanto à taxa de infiltração, a qual é dada pela derivada da equação 2 em relação ao tempo:

$\mathrm{i}=1 / 2 \mathrm{~S} \cdot \mathrm{t}^{-1 / 2}+\mathrm{A}$

No caso da parcela estudada, encontraram-se os seguintes valores médios de $\mathrm{S}$ e de $\mathrm{A}$ :

$\langle\mathrm{S}\rangle=0,520 \mathrm{~cm} / \mathrm{min}^{-1 / 2}$ e $\langle\mathrm{A}\rangle=0,484 \mathrm{~cm} / \mathrm{min}$.

O mapeamento dos parâmetros $\mathrm{S}$ e A é efetuado a seguir, pela técnica do Krigging. A mesma técnica é também utilizada para mapear a taxa percentual da fração de argila + silte e $\mathrm{K}_{0}$. Essa teoria é exposta em Delhome (1976) e é atualmente bastante utilizada em geociências. Distingue-se o Krigging simples quanto a dados que não apresentam derivas (correlações com posição geométrica) - o qual se baseia no ajustamento de um modelo ao semivariograma dos dados -, brutos e o Krigging universal, que permite a incorporação da deriva tal como mostrado nos semivariogramas filtrados. Em ambos os casos, o método permite levar em conta um eventual efeito de pepita.

$\mathrm{Na}$ Fig.5, foram mapeadas as variáveis $\%\left(\right.$ argila + silte), S, A e $\mathrm{K}_{0}$. Nota-se, de imediato, a forte correlação com o eixo $\mathrm{Y}$ dos valores de $\%(\mathrm{~A}+\mathrm{S})$. Para a taxa percentual da fração de argila + silte, foi considerado um modelo logarítmico, com deriva linear e um efeito de pepita, devido a uma 
microvariância de escala inferior a 15 m. Para a absorptividade, a técnica do Krigging simples foi adotada com um modelo linear simples sem efeito de pepita. Em relação às variáveis $\mathrm{A}$ e $\mathrm{K}_{0}$, foi adotado um modelo linear com deriva também linear e pepita, sendo o valor desta de 0,015 para a variável $\mathrm{A}$, e 0,013 para $\mathrm{K}_{0}$. Nos mapas relativos a $\mathrm{A}$ e $\mathrm{S}$, foram traçadas em linhas pontilhadas as isolinhas correspondentes aos valores médios: $\langle\mathrm{A}\rangle=0,484 \mathrm{e}$ $\langle\mathrm{S}\rangle=0,520$. A intercepção dessas linhas se dá entre os pontos de número 22 e 23 , que corresponderia ao ponto médio da parcela onde um teste de infiltração daria a taxa de infiltração média da parcela, assim como o volume infiltrado médio. O importante trabalho de Vachaud et al. (1985) mostra que um ponto médio do ponto de vista do teor em água no solo permanece médio ao longo do tempo, qualquer que seja este teor de água. Não existe na literatura outros trabalhos mostrando a estabilidade temporal das funções de distribuição das propriedades hidrodinâmicas do solo ou do teor de água presente nele. Os resultados deste trabalho mostram que em uma parcela agrícola, um estudo geoestatístico permite identificar o ponto médio da parcela quando se descreve o processo de infiltração pela equação de Philip. É importante salientar, aqui, que a equação de Philip é derivada, considerando uma carga constante à superfície durante o processo de infiltração, e é aplicada para tempos relativamente curtos. Por exemplo, não se adequa corretamente a uma infiltração relativa a uma precipitação, principalmente se a intensidade desta varia ao longo do tempo. No caso da equação de Green \& Ampt, se foi possível obter um mapa de $\mathrm{K}_{0}$ com o traçado da isolinha correspondente ao valor médio, a sucção na frente de umedecimento $\mathrm{h}_{\mathrm{f}}$ se mostrou extremamente sensível à variabilidade espacial, com um coeficiente de variação de $188,5 \%$ e $160 \%$, respectivamente, para os tempos de 30 e 90 minutos. Um ajustamento a uma distribuição de probabilidade desta variável não foi possível. Além do mais, aproximadamente na metade dos pontos onde testes de infiltração foram realizados, a infiltração não se comportou segundo as hipóteses de Green \& Ampt.

Esses resultados foram inferidos a partir do uso da equação de Philip. No caso de um processo mais complexo de infiltração como aqueles devidos à pre-
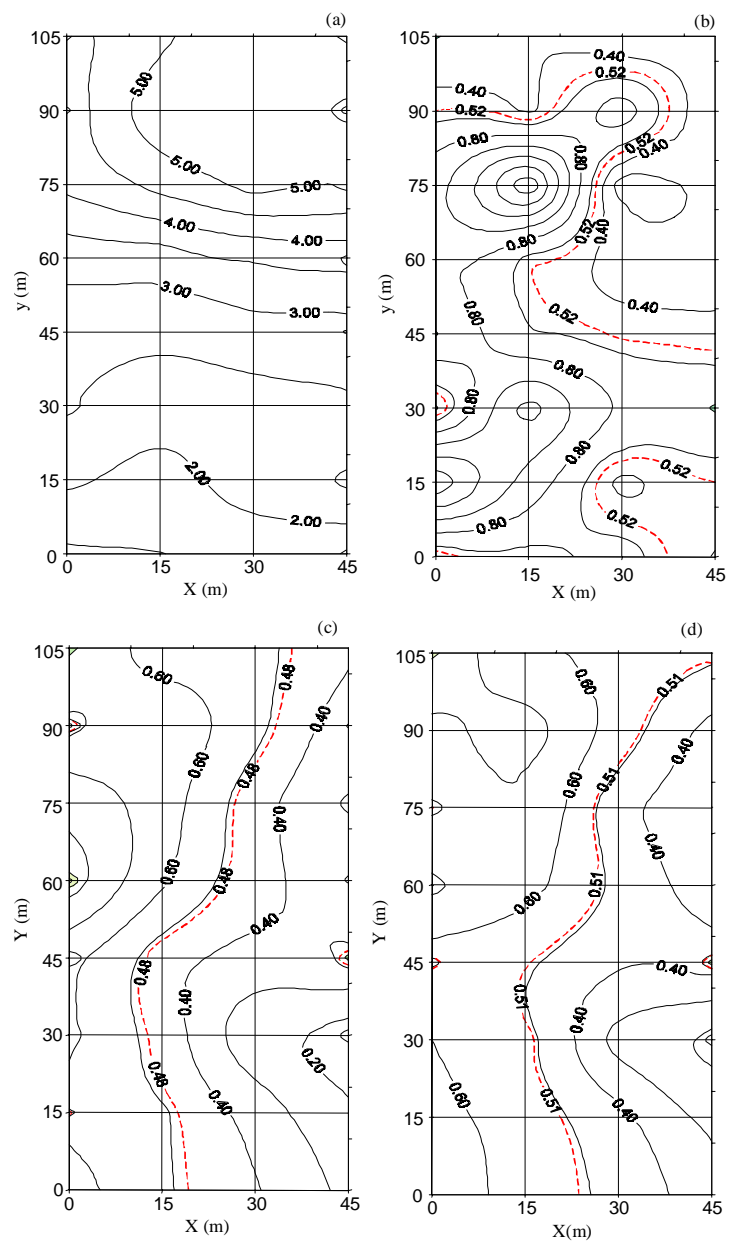

FIG. 5. Mapeamento pelo Krigging das variáveis:

(a) \% (argila + silte); (b) absortividade;

(c) constante da equação de Philip A;

(d) condutividade hidráulica.

cipitação, o uso da equação de Philip não é recomendado, preferindo-se, nesse caso, utilizar a equação de Green \& Ampt. Neste estudo, encontrou-se uma variabilidade muito grande do termo $h_{f}$, a pressão capilar na frente de umedecimento, utilizado na equação de Green \& Ampt. Considerando-se a nãolinearidade da equação de Green \& Ampt e a alta susceptibilidade à variabilidade espacial de $\mathrm{h}_{\mathrm{f}}$, torna-se legítimo perguntar se o uso da equação de Green \& Ampt em modelos hidrológicos distribuídos é válido. 


\section{CONCLUSÕES}

1. Em uma parcela relativamente pequena $\left(5.000 \mathrm{~m}^{2}\right)$ e de aparência homogênea, a variabilidade espacial é considerável; a análise estatística clássica permite caracterizar esta variabilidade.

2. As correlações entre textura, através da taxa percentual da fração de argila + silte, e os parâmetros hidrodinâmicos são insignificantes.

3. A análise geoestatística permite detectar a existência de uma certa estrutura ou grau de organização na distribuição espacial dos parâmetros estudados; a partir daí, pela técnica do Krigging, é possível mapear esses parâmetros e identificar, no campo, o local que representaria os valores médios do volume infiltrado.

\section{REFERÊNCIAS}

CARVALLO, H.O.; CASSEL, D.K.; HAMMOND, J.; BAUER, A. Spatial variability of in situ unsatured hydraulic conductivity of maddock sand loam. Soil Science Society of America. Journal, v.40, n.6, p.1-8, 1976.

CLAPP, R.; HORNBERGER, G. Empirical equations for some hydraulic properties. Water Resources Research, v.14, n.4, p.601-604, 1978.

DELHOME, J.P. Applications de la théorie des variables régionalisées dans les sciences de l'eau. Paris: Université Pierre et Marie Curie, 1976.130p. Thèse de Docteur.

IMBERNON, J. Variabilité spatiale des caractéristiques hydrodinamiques d'un sol du Sénégal. Grenoble: L'Institut National Polytechnique de Grenoble, 1981. 153p. Thèse de Docteur.

LIMA, C.A.G. Estudo da variabilidade espacial da infiltração: aspectos teóricos experimentais. João Pessoa: Universidade Federal da Paraíba, 1992. 142p. Dissertação de Mestrado.

LUXMOORE, R.J.; SHARMA, M.L. Runnof responses to soil heterogeneity: experimental and simulation comparisons for two contrasting watersheds. Water Resources Research, v.16, n.4, p.675-684, 1980.
MILLER, E.E.; MILLER, R.D. Physical theory for capilary flow phenomena. Journal of Applied Physics, v.24, p.324-332, 1956.

NIELSEN, D.R.; BIGGAR, J.W.; ERH, K.T. Spatial variability of field measured soil-water properties. Hilgardia, v.3, n.42, p.215-259, 1973.

ROGOWSKY, A.S. Watershed physical soil variability criteria. Water Resources Research, v.8, n.4, p.1015-1023, 1972.

RUSSO, D.; BRESLER, E. Field determination of soil hydraulic properties for statistical analysis. Soil Science Society of America. Journal, v. 44, n.5, p.697-702, 1980.

RUSSO, D.; BRESLER, E. Soil hydraulic properties as stochastic processes. Soil Science Society of America. Journal, v.45, p.682-687, 1981.

SHARMA, M.L; GANDER, A.; HUNT, C.G. Soil spatial variability of infiltration in a watershed. Journal of Hydrology, v.45, n.12, p.101-122, 1980.

SHARMA, M.L.; LUXMOORE, R.J. Soil spatial variability and its consequences on simulated water balance. Water Resources Research, v.15, n.5, p.1567-1573, 1979.

SISSON, J.B.; WIERENGA, P.J. Spatial variability of steady-state infiltration rates as a stochastic process. Soil Science Society of America. Journal, v.45, n.5, p.699-704, 1981.

VACHAUD, G.; SILANS, A.P.; BALABANIS, P.; VAUCLIN, M. Temporal stability of measured soil water probability densy function. Soil Science Society of America. Journal, v.49, n.2, p.822-828, 1985.

VAUCLIN, M.; VACHAUD, G.; IMBERNON, J. Spatial variability of some soil physical properties over one-hectare field plot. Colorado: Fort-collins, 1981. 50p.

VIEIRA, S. R.; NIELSEN, D.R.; BIGGAR, J.W. Spatial variability of field. Measured Infiltration Rate. Soil Science Society of America. Journal, v.45, n.4, p.1040-1048, 1981.

WARRICK, A.W.; MULLER, G.J.; NIELSEN, D.R. Prediction of the soil water flux based upon field measured soil-water properties. Soil Science Society of America. Journal, v.41, n.5, p.4-19, 1977. 